\title{
Conditional loyalty and its implications for pricing
}

\author{
Massimo A. De Francesco ${ }^{1}$ (D)
}

Received: 17 January 2021 / Accepted: 17 August 2021 / Published online: 27 September 2021

(c) The Author(s) 2021

\begin{abstract}
Bertrand-Edgeworth competition has recently been analyzed under imperfect buyer mobility, as a game in which, once prices are chosen, a static buyer subgame (BS) is played where the buyers choose which seller to visit (see, e.g., Burdett et al. in J Political Econ 109:1060-1085, 2001). Our paper considers a symmetric duopoly where two buyers play a two-stage BS of imperfect information after price setting. An "assessment equilibrium" of the BS is shown to exist in which, with prices at the two firms sufficiently close to each other, the buyers keep loyal if previously served. Conditional loyalty is proved to increase the duopolists' market power: at the corresponding subgame perfect equilibrium of the entire game, the uniform price is higher than that corresponding to the equilibrium of the BS in which the buyers are persistently randomizing.
\end{abstract}

Keywords Bertrand-Edgeworth competition · Matching · Imperfect buyer mobility · Conditional loyalty $\cdot$ Assessment equilibrium

JEL Classification D430 $\cdot$ L130

\section{Introduction}

In models of Bertrand-Edgeworth competition with homogeneous product it has customarily been taken for granted that no firm is selling below capacity while an equally expensive firm is facing excess demand or some positive quantity is sold by a more expensive firm. Such mismatchings would in fact be immediately ruled out if the buyers could instantly and costlessly move across sellers. Quite differently, some models have incorporated imperfect buyer mobility by letting the buyers visit just one seller in the buyer subgame (BS) that is played after price decisions are made (see Peters 1984 and 2000; Deneckere and Peck 1995; Burdett et al. 2001). Then,

Massimo A. De Francesco

defrancesco@unisi.it

1 Department of Economics and statistics, University of Siena, Piazza S. Francesco 7,

53100 Siena, Italy 
even if total capacity could meet total demand, the buyer's payoff would depend on the perceived service probability as well as the price to be paid if served. Even with relatively few buyers, there are many pure strategy equilibria (PSEs) of the BS when prices at the different sellers are sufficiently close to each other: thus, ever since Peters (1984), the attention has understandably been focused on the (symmetric) mixed strategy equilibrium (MSE) of the BS, where mismatchings of the aforementioned type arise with positive probability. But then, lack of ex-post buyer mobility significantly affects pricing. To see this, consider the following setting. A number of identical firms produce on demand a homogeneous good, at constant unit cost up to their fixed capacity. Each identical buyer demands a fixed quantity, at any price not higher than the reservation price, and total demand is equal to total capacity. As in Burdett et al. (2001), the firms set prices whereupon each buyer makes his decision. Unlike with perfect mobility, equilibrium prices are less than the reservation price: in fact, with uniform prices, expected output is less than each firm's capacity at the MSE of the BS; consequently, with the rival firms charging the reservation price it pays to undercut since all buyers would then try the cheapest firm.

Subsequent contributions to this literature on pricing and "directed" search allow for an influence of realized demand on prices. In Camera and Selcuk (2009), the profile of prices posted by the sellers determines the probability distribution of the buyers across the sellers; imperfect commitment to the posted price may leave room to renegotiating the price in light of actual demand. In Geromichalos (2014), each seller specifies how his own price will depend on his forthcoming demand; based on these price schedules, a mixed strategy equilibrium obtains. In either case, differences in prices at equilibrium are derived in terms of different pressure of demand over capacity at the various sellers. Another recent innovation in this literature is contained in Shi (2016), where a multi-stage model is developed in which, in each stage, the firms announce prices as well as any service priority they might offer to loyal buyers while the buyers, based on this information and the history of previous matchings at the various firms, choose the probability of visiting any seller.

In this paper we address Bertrand-Edgeworth competition with imperfect buyer mobility along an alternative approach, seemingly not in contrast with what is often observed in reality. It is assumed below that a certain good is demanded repeatedly by each buyer over a certain period in which prices are fixed and that, each time the demand decision is made, the buyer also chooses which seller to visit in search for the good. Also, we assume that, when making this choice, the buyer does not have perfect information about pressure of demand on capacity at the various sellers in the recent past; at the same time, she can make some inference in this respect, based on her personal experience with the seller(s) she visited in the past. A previous inquiry on buyers' incentives in such a context is in De Francesco (2005), limited to the case in which prices are exogenously given and equal at the existing sellers. It was shown that, in a dynamic buyer game in which each buyer repeatedly chooses which seller to try, there is scope for the emergence of a norm of "conditional loyalty", according to which each buyer keeps loyal to the seller previously chosen conditional on having been served, while switching to any seller not yet tried before if previously rationed. More specifically, it was shown that, if all the buyers are conditionally loyal, then, when total industry capacity is enough to meet total demand, mismatchings between demand and supply at the seller's level will 
disappear with unit probability in a finite number of stages of the buyer game. Not only this, but conditional loyalty was proven to be an "assessment equilibrium" in the twoseller case, again, taking prices as exogenously equal.

The present contribution purports to further develop that approach. The scope for conditional loyalty will here be analyzed in a setup in which prices might differ at the various sellers, which in its turn will allow us to see the implications of conditional loyalty for price determination in the first place. We construct a very simple model to deal with buyers' imperfect mobility and information in a dynamic setting: we assume there are just two sellers and two buyers; after prices are set by the duopolists, a two-stage BS is assumed to be played where, in each stage, each buyer chooses independently which seller to visit with no direct knowledge, when playing the second stage, of the choice made by the other buyer in the first stage. In such a model, the aforementioned coordination problem affecting the PSEs of the static BS might appear to be less severe than with several buyers and sellers; yet, the problem is still there and, furthermore, the two-seller, two-buyer, two-stage BS greatly simplifies the analysis of the dynamic BS and hence of pricing. Here are the main findings that, in our view, provide helpful insights for more general settings. Two alternative types of equilibria of the dynamic BS are characterized, leading to different predictions for BSs in which prices are sufficiently close to each other. In one equilibrium, the buyers' beliefs on previous matchings at the firms are irrelevant to their moves in the second stage of the BS inasmuch as the MSE of the static BS is played repeatedly. But another equilibrium exists, in which the beliefs of the buyers on previous matches play a key role. In this "assessment equilibrium", so long as prices at the two sellers are sufficiently close, in the second stage each buyer keeps loyal to the seller previously chosen if served, while moving to the other seller if rationed: on the corresponding equilibrium path, both buyers are served in the second stage of the BS. It deserves to be emphasized that the driving force behind conditional loyalty is just the strategic interaction among the buyers under imperfect information: if the other buyer is expected to keep loyal when served, then being conditionally loyal maximizes one's own service probability. Most importantly, under the assumptions of the model of the present paper, the pattern of conditional loyalty reduces the incentive to undercut the rival's price: at the (subgame-perfect) equilibrium of the entire game, the uniform price is higher than it would be under persistent randomization on the part of the buyers.

The rest of the paper is organized as follows. To prepare the ground for our positive contribution, "Pricing under a static buyer subgame" recounts, with a few additional qualifications, Burdett et al.'s two-seller two-buyer version (2001, pp. 1062-1067) of their more general model of price determination under a static BS. "Pricing under a dynamic buyer subgame" develops our model of duopoly price determination in which two buyers are playing a two-stage BS of imperfect information, in each stage choosing which seller to visit. The last section briefly concludes.

\section{Pricing under a static buyer subgame}

Two identical and risk-neutral firms, $A$ and $B$, sell a homogeneous and indivisible commodity to two identical and risk-neutral buyers, $h$ and $k$. When no ambiguity can arise, we refer to the generic buyer as buyer $h$. Each firm $i \in\{A, B\}$ has productive 
capacity $\bar{y}_{i}=1$ and independently announces its own price $p_{i}$; the chosen price pair identifies a $\mathrm{BS}$, in the following referred to as a " $\left(p_{A}, p_{B}\right)-\mathrm{BS}$ ". In this section we analyze static $\left(p_{A}, p_{B}\right)$-BSs, in which any buyer $h$ demands one unit of the commodity if $\min \left\{p_{A}, p_{B}\right\} \leq 1$ - where 1 is the buyer's reservation price-and chooses a probability distribution $\left(v_{h}, 1-v_{h}\right)$ for his visit to sellers $A$ and $B$, respectively. For brevity, we denote by $\left(v_{h}, v_{k}\right)$, rather than by $\left(\left(v_{h}, 1-v_{h}\right),\left(v_{k}, 1-v_{k}\right)\right)$, the profile of probability distributions. Firm $i$ costlessly produces quantity $y_{i}$, the smaller of its forthcoming demand and its capacity.

For the sake of comparison, it is worth to preliminarily look at the polar case of perfect mobility of buyers. In that case, with, say, $p_{B}<p_{A} \leq 1$, it pays for each buyer to try seller $B$ in the first place since, if rationed, the buyer would still be able to purchase the commodity by quickly moving to seller $A$. Consequently, charging the buyer's reservation price is for each seller a strictly dominant strategy since, no matter $p_{B}$, seller $A$ would then sell its capacity output for any $p_{A} \leq 1{ }^{1}$

Coming back to our setting in which the buyer can visit just one seller in the static $\mathrm{BS}$, we will take it for granted that ${ }^{2}$

$$
p_{i} \in[0 ; 1], \quad i \in\{A, B\} .
$$

We denote by $\mathcal{E}_{h}$ the set of all possible events that buyer $h$ may face and by $\mathbf{e}_{h}$ its generic element: $\mathbf{e}_{h} \in \mathcal{E}_{h}=\left\{A s_{h}, A r_{h}, B s_{h}, B r_{h}\right\}$, where, for instance, $A s_{h}$ (resp. $A r_{h}$ ) is the event of buyer $h$ visiting seller $A$ and being served (resp. rationed) there. With both buyers at seller $i$, each of them is served with probability $1 / 2$. We denote by $\pi\left(\mathbf{e}_{h}\left(v_{h}, v_{k}\right)\right)$ the probability of the event $\mathbf{e}_{h}$ under some profile $\left(v_{h}, v_{k}\right)$ of probability distributions; and, for brevity, for any given price pair $\left(p_{A}, p_{B}\right)$, we denote by $u_{h}\left(v_{h}, v_{k}\right)$, rather than by $u_{h}\left(v_{h}, v_{k} ; p_{A}, p_{B}\right)$, buyer $h$ 's payoff (expected surplus) under the profile of probability distributions $\left(v_{h}, v_{k}\right)$. Clearly,

$$
\begin{aligned}
u_{h}\left(v_{h}, v_{k}\right) & =v_{h} \pi\left(A s_{h}\left(1, v_{k}\right)\right)\left(1-p_{A}\right)+\left(1-v_{h}\right) \pi\left(B s_{h}\left(0, v_{k}\right)\right)\left(1-p_{B}\right) \\
& =v_{h}\left(\frac{v_{k}}{2}+1-v_{k}\right)\left(1-p_{A}\right)+\left(1-v_{h}\right)\left(v_{k}+\frac{1-v_{k}}{2}\right)\left(1-p_{B}\right) .
\end{aligned}
$$

With $p_{A}$ and $p_{B}$ sufficiently close to each other, the BS has a symmetric equilibrium $\left(v_{h}, v_{k}\right)=(\widetilde{v}, \widetilde{v})$ : for $v_{k}=\widetilde{v}$, buyer $h$ is indifferent between visiting either seller, hence $\widetilde{v}$ is the solution of the equation

$$
\left(\frac{v}{2}+1-v\right)\left(1-p_{A}\right)=\left(v+\frac{1-v}{2}\right)\left(1-p_{B}\right)
$$

namely,

$$
\widetilde{v}=\widetilde{v}\left(p_{A}, p_{B}\right)=\frac{1-2 p_{A}+p_{B}}{2-p_{A}-p_{B}} .
$$

The equilibrium $\left(v_{h}, v_{k}\right)=(\widetilde{v}, \widetilde{v})$ exists if and only if

\footnotetext{
1 The buyer is assumed to purchase the good, when indifferent between purchasing or not.

2 As will be argued below, one can actually take it for granted that $p_{i} \in(0 ; 1)$.
} 


$$
2 p_{B}-1 \leq p_{A} \leq \frac{1+p_{B}}{2}
$$

Note that the interval $\left[2 p_{B}-1, \frac{1+p_{B}}{2}\right]$ exists if $p_{B} \leq 1$ and degenerates to 1 if $p_{B}=1$. If system (5) holds and $p_{B}<1$, then $p_{A}<1$ and, more thoroughly: if

$$
2 p_{B}-1<p_{A}<\frac{1+p_{B}}{2}
$$

then

$$
\widetilde{v}\left(p_{A}, p_{B}\right) \in(0,1)
$$

namely, the symmetric equilibrium is in mixed strategies; if ${ }^{3}$

$$
2 p_{B}-1=p_{A}<\frac{1+p_{B}}{2},
$$

then

$$
\widetilde{v}\left(p_{A}, p_{B}\right)=1
$$

if

$$
2 p_{B}-1<p_{A}=\frac{1+p_{B}}{2}
$$

then

$$
\widetilde{v}\left(p_{A}, p_{B}\right)=0 \text {. }
$$

Finally, if system (5) holds and $p_{B}=1$, then $p_{A}=1$. For definiteness, we let

$$
\widetilde{v}(1,1)=\frac{1}{2} \text {. }
$$

Holding system (6), two asymmetric pure strategy equilibria (PSEs) also exist, $\quad\left(v_{h}, v_{k}\right)=(1,0)$ and $\left(v_{h}, v_{k}\right)=(0,1)$, yielding payoffs $1-p_{A}$ and $1-p_{B}$ to the buyers. Either PSE Pareto-dominates the mixed strategy equilibrium (MSE) $\left(v_{h}, v_{k}\right)=(\widetilde{v}, \widetilde{v})$ : indeed, taking into account Eq. (3), $u_{h}(\widetilde{v}, \widetilde{v})=\frac{2-\widetilde{v}}{2}\left(1-p_{A}\right)=\frac{\widetilde{v}+1}{2}\left(1-p_{B}\right)<\min \left\{1-p_{A}, 1-p_{B}\right\}$. On the other hand, in order for the buyers to play any such PSE they should somehow coordinate ex-ante their moves in the game. This is problematic since pre-play communication might be unfeasible or quite costly to the buyers. Furthermore, a major difficulty in agreeing

\footnotetext{
$\overline{{ }^{3} \text { Note that if } 2 p_{B}-1=p_{A} \text {, then }} p_{B} \geq \frac{1}{2}$ since $p_{i} \geq 0$ (see condition (1)).
} 
to play a PSE arises on distributive grounds whenever $p_{A} \neq p_{B}$, since then each buyer obviously prefers the PSE in which he visits the cheaper seller. ${ }^{4}$

Finally, with $p_{B}<1$, playing $v_{h}=0$ (resp. $\left.v_{h}=1\right)$ is dominant if $p_{A} \geq\left(1+p_{B}\right) / 2$ (resp. $p_{A} \leq 2 p_{B}-1$ )—strictly dominant if the inequality is strict.

Based on all the above, for any price pair $\left(p_{A}, p_{B}\right) \in[0,1]^{2}$ we assume that the symmetric equilibrium $\left(v_{h}, v_{k}\right)=\left(v^{*}, v^{*}\right)$ of the $\mathrm{BS}$ is played, where ${ }^{5}$

$$
v^{*}=v^{*}\left(p_{A}, p_{B}\right)= \begin{cases}1 & \text { if } p_{A}<2 p_{B}-1 \\ \widetilde{v} \in[0,1] & \text { if } 2 p_{B}-1 \leq p_{A} \leq \frac{1+p_{B}}{2} \\ 0 & \text { if } p_{A}>\frac{1+p_{B}}{2} .\end{cases}
$$

and $\widetilde{v}=\widetilde{v}\left(p_{A}, p_{B}\right)$ (see Eq. (4)). The function $v^{*}=v^{*}\left(p_{A}, p_{B}\right)$ presents a discontinuity at $\left(p_{A}, p_{B}\right)=(1,1): v^{*}(1,1)=1 / 2$ (see Eq. (12)) whereas $\lim _{p_{A} \nearrow 1} v^{*}\left(p_{A}, 1\right)=1$. We denote by $E y_{i}(v)(i=A, B)$ firm $i$ 's expected output for any profile $\left(v_{h}, v_{k}\right)=(v, v)$ : clearly,

$$
E y_{A}(v)=v^{2}+2 v(1-v)
$$

and

$$
E y_{B}(v)=(1-v)^{2}+2 v(1-v) .
$$

Firm $A$ 's expected output at the symmetric equilibrium of some $\left(p_{A}, p_{B}\right)$-BS, is written $E y_{A}^{*}\left(p_{A}, p_{B}\right)=E y_{A}\left(v^{*}\left(p_{A}, p_{B}\right)\right)$. We can safely let

$$
p_{i} \in(0,1), \quad i \in\{A, B\},
$$

since $p_{A}=1$ and $p_{A}=0$ are never-best responses when $\left(v_{h}, v_{k}\right)=\left(v^{*}, v^{*}\right)$. In fact, firm $A$ 's payoff (expected profit) at the symmetric equilibrium of any $\left(p_{A}, p_{B}\right)$-BS is $E \Pi_{A}^{*}\left(p_{A}, p_{B}\right)=p_{A} E y_{A}\left(v^{*}\left(p_{A}, p_{B}\right)\right)$ and therefore: if $p_{B}<1$, then $\left[E \Pi_{A}^{*}\left(p_{A}, p_{B}\right)\right]_{p_{A} \in\left(0, \frac{1+p_{B}}{2}\right)}>E \Pi_{A}^{*}\left(1, p_{B}\right)=0$ (see Eqs. (4), (6), (13) and (14)); if $p_{B}=1$, then $\lim _{p_{A} \nearrow 1} E \Pi_{A}^{*}\left(p_{A}, 1\right)=1>E \Pi_{A}^{*}(1,1)=3 / 4$ (see Eqs. (13), (14), and (12)).

Conditional on the equilibrium $\left(v_{h}, v_{k}\right)=\left(v^{*}, v^{*}\right)$ to be played in any $\mathrm{BS}$, the rate of change of firm $A$ 's expected profit with respect to its own strategy (henceforth, firm $A$ 's marginal payoff) is

$$
\frac{\partial E \Pi_{A}^{*}\left(p_{A}, p_{B}\right)}{\partial p_{A}}=E y_{A}\left(v^{*}\right)+\left.p_{A} \frac{d E y_{A}(v)}{d v}\right|_{v=v^{*}} \frac{\partial v^{*}\left(p_{A}, p_{B}\right)}{\partial p_{A}} .
$$

\footnotetext{
${ }^{4}$ Such distributive dispute could only be overcome if the buyers were able to agree upon some side payment between them.

5 Taking into account Eqs. (9) and (11)), it should be noted that, for $p_{B}<1$ and $p_{A}=2 p_{B}-1$ (resp. $\left.p_{A}=\left(1+p_{B}\right) / 2\right)$, the second line of Eq. (13) selects the profile of weakly dominant strategies $\left(v_{h}, v_{k}\right)=(\widetilde{v}, \widetilde{v})=(1 ; 1)\left(\operatorname{resp} .\left(v_{h}, v_{k}\right)=(\widetilde{v}, \widetilde{v})=(0 ; 0)\right)$, out of the continuum of equilibria $\left(v_{h}, v_{k}\right)=\left(\widetilde{v}, v_{k}\right)$ (any $v_{k} \in[0 ; 1]$ ).
} 
Holding system $(6), v^{*}=\widetilde{v} \in(0,1),\left.\frac{d E y_{A}(v)}{d v}\right|_{v=\widetilde{v}}=2(1-\widetilde{v})>0,\left.\frac{d^{2} E y_{A}(v)}{d v^{2}}\right|_{v=\widetilde{v}}=-2$, $\frac{\partial \widetilde{v}\left(p_{A}, p_{B}\right)}{\partial p_{A}}=\frac{-3\left(1-p_{B}\right)}{\left(2-p_{A}-p_{B}\right)^{2}}<0$, and $\frac{\partial^{2} \widetilde{v}\left(p_{A}, p_{B}\right)}{\partial p_{A}^{2}}=\frac{-6\left(1-p_{B}\right)}{\left(2-p_{A}-p_{B}\right)^{3}}<0$. All this implies strict concavity of $E \Pi_{A}^{*}\left(p_{A}, p_{B}\right) \quad$ in $\quad p_{A}: \quad \frac{\partial^{2} E \Pi_{A}^{*}\left(p_{A}, p_{B}\right)}{\partial p_{A}^{A}}=\left.2 \frac{d E y_{A}(v)}{d v}\right|_{v=\widetilde{v}} \frac{\partial \widetilde{v}\left(p_{A}, p_{B}\right)}{\partial p_{A}}+\left.p_{A} \frac{d^{2} E y_{A}(v)}{d v^{2}}\right|_{v=\widetilde{v}}\left(\frac{\partial \widetilde{v}\left(p_{A}, p_{B}\right)}{\partial p_{A}}\right)^{2}$ $+\left.p_{A} \frac{d E y_{A}(v)}{d v}\right|_{v=\widetilde{v}} \frac{\partial^{2} \widetilde{v}\left(p_{A}, p_{B}\right)}{\partial p_{A}^{2}}<0$. (Of course, a similar argument would prove strict concavity of $E \Pi_{B}^{*}\left(p_{A}, p_{B}\right)$ in $p_{B}$. $)$ At a symmetric equilibrium of the price game, $\left(p_{A}, p_{B}\right)=(p, p)$ and $\quad \widetilde{v}=1 / 2$. As can easily be verified, $\left.\frac{\partial E \Pi_{A}^{*}\left(p_{A}, p_{B}\right)}{\partial p_{A}}\right|_{\left(p_{A}, p_{B}\right)=(p, p)}=\left.\frac{\partial E \Pi_{B}^{*}\left(p_{A}, p_{B}\right)}{\partial p_{B}}\right|_{\left(p_{A}, p_{B}\right)=(p, p)}$, so that, without loss of generality, we can focus on firm $A$ 's profit maximization. Since $\left[E y_{A}(v)\right]_{v=\frac{1}{2}}=\frac{3}{4},\left.\frac{d E y_{A}(v)}{d v}\right|_{v=\frac{1}{2}}=1$ and $\left.\frac{\partial v^{*}\left(p_{A}, p_{B}\right)}{\partial p_{A}}\right|_{\left(p_{A}, p_{B}\right)=(p, p)}=\frac{-3}{4(1-p)}$, from the first-order condition $\left.\frac{\partial E \Pi_{A}^{*}\left(p_{A}, p_{B}\right)}{\partial p_{A}}\right|_{\left(p_{A}, p_{B}\right)=(p, p)}=0$ for a best response it is obtained $p=1 / 2$. This stands in stark contrast with the case of perfect mobility, where charging $p_{i}=1$ (each $i \in\{A, B\}$ ) is a strictly dominant strategy.

\section{Pricing under a dynamic buyer subgame}

In this section, it is assumed that, after price setting at stage $t=0$, a two-stage BS of imperfect information is played: in each stage $t=1,2$ of the $\left(p_{A}, p_{B}\right)-\mathrm{BS}$, each buyer $h$ demands one unit of the (perishable) commodity and chooses the probability distribution $\left(v_{h, t}, 1-v_{h, t}\right)$ for his visit to sellers $A$ and $B$. Again, we take it for granted that $p_{i} \in[0 ; 1](i \in\{A, B\})$. For brevity, we denote by $\left(v_{h, t}, v_{k, t}\right)$-rather than by $\left(\left(v_{h, t}, 1-v_{h, t}\right),\left(v_{k, t}, 1-v_{k, t}\right)\right)$ - the profile of stage- $t$ probability distributions. In stage $t$, firm $i$ produces quantity $y_{i, t}$, the lower of its forthcoming demand in $t$ and its capacity $\bar{y}_{i}=1$. We denote by $E y_{i, t}(v)$ firm $i$ 's stage- $t$ expected output under the symmetric profile $\left(v_{h, t}, v_{k, t}\right)=(v, v)$ of probability distributions. It is assumed that each buyer maximizes the undiscounted sum of his expected surplus over the BS and that each firm maximizes the undiscounted sum of its expected profits. We denote by $\mathcal{E}_{h, t}=\left\{\mathbf{e}_{h, t}\right\}=\left\{A s_{h, t}, A r_{h, t}, B s_{h, t}, B r_{h, t}\right\}$ the set of stage- $t$ possible events buyer $h$ may face and by $\pi\left(\mathbf{e}_{h, t}\left(v_{h, t}, v_{k, t}\right)\right)$ the probability of the event $\mathbf{e}_{h, t}$ under the profile $\left(v_{h, t}, v_{k, t}\right)$.

With both buyers at seller $i$ in $t=2$, each buyer is assumed to be served with probability $1 / 2$, regardless of which buyer that seller served in $t=1$. As clarified below, to choose $v_{h, 2}$ in $t=2$ it might make sense to buyer $h$ to make an inference on $\mathbf{e}_{k, 1} \in \mathcal{E}_{k, 1}=\left\{A s_{k, 1}, A r_{k, 1}, B s_{k, 1}, B r_{k, 1}\right\}$ from his information set $I_{h}=\left(\mathbf{e}_{h, 1},\left(p_{A}, p_{B}\right)\right)$ -henceforth, $I_{h}=\left(\mathbf{e}_{h, 1}, \cdot\right)$, for brevity - and his conjecture on $k$ 's previous move.

A behavioral strategy-call it $\Theta_{h}$-for buyer $h$ is a pair of functions $\Theta_{h}=\left(v_{h, 1}=v_{h, 1}\left(p_{A}, p_{B}\right), v_{h, 2}=v_{h, 2}\left(I_{h}\right)\right)$ : the probability distribution for $t=1$ is a function of prices while the probability distribution for $t=2$ depends on prices and the experience being made by the buyer in $t=1$. We denote by $\Theta_{h \mid 2}$ any strategy containing the same function $v_{h, 2}=v_{h, 2}\left(I_{h}\right)$ for $t=2$ as $\Theta_{h}$ does. 
For brevity, we denote by $U_{h}\left(\Theta_{h}, \Theta_{k}\right)$, rather than by $U_{h}\left(\Theta_{h}, \Theta_{k} ; p_{A}, p_{B}\right)$, buyer $h$ 's ex ante payoff, under strategy profile $\left(\Theta_{h}, \Theta_{k}\right)$, over some specified $\left(p_{A}, p_{B}\right)$-BS: $U_{h}\left(\Theta_{h}, \Theta_{k}\right)=\sum_{t=1}^{2} u_{h, t}\left(\Theta_{h}, \Theta_{k}\right)$, where $u_{h, t}\left(\Theta_{h}, \Theta_{k}\right)(t=1,2)$ is buyer $h$ 's stage- $t$ expected surplus, as valued at the onset of the BS. Furthermore, we denote by $u_{h, t}\left(v_{h, t}, \Theta_{k}\right)$ buyer $h$ 's ex-ante stage- $t$ expected surplus, when buyer $h$ chooses the probability distribution $\left(v_{h, t}, 1-v_{h, t}\right)$ and buyer $k$ adheres to strategy $\Theta_{k}$.

We now look at the equilibria of the dynamic BSs. In $\left(p_{A}, p_{B}\right)$-BSs in which system (6) does not hold and $p_{i}<1$ for some $i \in\{A, B\}$, the buyers are playing in each stage the dominant strategy of the static BS: $\left(v_{h, t}, v_{k, t}\right)=(1,1)$ if $p_{A} \leq 2 p_{B}-1$ and $\left(v_{h, t}, v_{k, t}\right)=(0,0)$ if $p_{A} \geq\left(1+p_{B}\right) / 2$.

Turning to $\left(p_{A}, p_{B}\right)$-BSs in which system (6) holds, one type of equilibria is such that an equilibrium of the static BS is played already from the first stage. For instance, there are equilibria in which one of the two asymmetric PSEs of the static BS- $\left(v_{h, t}, v_{k, t}\right)=(1,0)$ or $\left(v_{h, t}, v_{k, t}\right)=(0,1)$-is played in each stage. But, again, we rule out the event of the buyers playing any such equilibrium by assuming that pre-play communication is too costly or unfeasible. So, within that type of equilibria, we only remain with equilibria in which the buyers persistently randomize over the BS when prices are close enough so as to meet system (6). This leads to the following proposition, whose proof derives straightforwardly from the arguments in the previous section.

Proposition 1 Let $\Theta_{h}^{*}=\left(v^{*}, v^{*}\right)$ and $\Theta_{k}^{*}=\left(v^{*}, v^{*}\right)$, where $v^{*}$ is defined by Eq. (13). The strategy profile $\left(\Theta_{h}^{*}, \Theta_{k}^{*}\right)$ induces a Nash equilibrium in each $\left(p_{A}, p_{B}\right)-B S$.

Once again, in each $\left(p_{A}, p_{B}\right)$-BS in which prices meet system (6) the equilibrium induced by the strategy profile $\left(\Theta_{h}^{*}, \Theta_{k}^{*}\right)$ is inefficient. For instance, with $p_{A}=p_{B}=p$, we have $v^{*}=\frac{1}{2}$ : hence, in each stage each buyer is served with a probability of $\frac{3}{4}$ and each buyer's equilibrium payoff over the two-stage BS is $(1-p) \frac{3}{4} \times 2$, whereas by repeatedly playing a PSE of the static BS each buyer would get $(1-p) \times 2$ over the two-stage BS.

An interesting question is whether equilibria of a different type exist, in which the experience undergone by buyer $h$ in $t=1$ will affect $h$ 's prediction on buyer $k$ 's move in $t=2$, thereby affecting $h$ 's move in $t=2$. Equilibria of this type do exist: in particular, as will be shown later on, there are equilibria in which the following norm of "conditional loyalty" (CL) is adhered to when system (6) holds.

Definition 1 According to CL, if previously served a buyer will visit in $t=2$ the same seller he visited in $t=1$, while visiting the other seller if previously rationed.

Before incorporating the norm of CL into a candidate equilibrium strategy, it is important to point out that such a norm is an effective ex-post coordination device among the buyers. 
Proposition 2 Under $C L$, both buyers are certainly served in $t=2$; in contrast, a unilateral deviation from CL results in each buyer being rationed with positive probability.

Proof Suppose that, in $t=1$, buyer $h$ is served and buyer $k$ is rationed by $A$ or that $h$ is served by $A$ and $k$ is served by $B$. Then, under CL, $\left(v_{h, 2}, v_{k, 2}\right)=(1,0)$ and hence both buyers will be served in $t=2$. If, instead, buyer $h$ unilaterally deviates, then $v_{h, 2}<1$ and hence service probability will be $v_{h, 2}+\frac{1-v_{h, 2}}{2}<1$ for each; if buyer $k$ deviates, then $v_{k, 2}>0$, implying service probability $\frac{v_{k, 2}}{2}+1-v_{k, 2}<1$ for each.

Remark 1 The effectiveness of CL as an ex-post coordination device in a dynamic BS was already pointed out in Goldman et al. (2004), through a computational model with automated buyers and sellers, and in De Francesco (1998 and 2005), in a context in which there are $m$ buyers, each of them demanding one unit of a commodity and choosing in each stage which of $n$ sellers to visit, each of the latter endowed, as in our twobuyer and two-seller model, with capacity $\bar{y}_{i}=m / n$, where $m / n$ is an integer. Unlike in our present model, in De Francesco $(1998,2005)$ the focus was on BSs in which prices are exogenously given and the same at all the sellers. As for the static BS, quite intuitively, the set of PSEs is made up of any profile of deterministic choices resulting in $\mathrm{m} / \mathrm{n}$ visitors for each seller — which, by the way, represents a perfect matching of buyers and sellers (see Proposition 1 in De Francesco 2005). But, of course, there are lots of such equilibria: ${ }^{6}$ thus, in view of the underlying coordination problem already emphasized in our two-seller and two-buyer setup, within the context of a static BS one is understandably led to look at the MSE, in which each buyer visits any seller with a probability of $1 / n$. At the MSE, the demand $d_{i}$ forthcoming to any firm $i$ is clearly a binomial random variable, with probability distribution $\operatorname{Pr}\left(d_{i}, m, \frac{1}{n}\right)=\left(\begin{array}{c}m \\ d_{i}\end{array}\right)\left(\frac{1}{n}\right)^{d_{i}}\left(1-\frac{1}{n}\right)^{m-d_{i}}$ $\left(d_{i}=0, \ldots, m\right)$. Since total demand equals total capacity and all the firms are visited with the same probability by each buyer, expected demand $E d_{i}$ for each firm is equal to its capacity $m / n .^{7}$ At the same time, expected output $E y_{i}$ is less than capacity: indeed, while output falls short of capacity if the number of buyers $d_{i}$ visiting the firm is less than $m / n$, output is just equal to capacity if $d_{i}>m / n$. Thus, each firm's expected output per unit of capacity, $\frac{E y_{i}}{\bar{y}_{i}}$ — equivalently, the probability for each buyer to be served-will be lower than 1 . On reflection,

$$
\frac{E y_{i}}{\bar{y}_{i}}=\frac{\sum_{d_{i}=0}^{m}\left(\begin{array}{c}
m \\
d_{i}
\end{array}\right)\left(\frac{1}{n}\right)^{d_{i}}\left(1-\frac{1}{n}\right)^{m-d_{i}} \min \left\{d_{i}, \frac{m}{n}\right\}}{m / n} .
$$

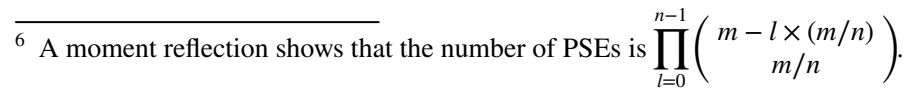

${ }^{7}$ More formally, from $d_{1}+\ldots+d_{n}=m$ it follows that $E d_{1}+\ldots+E d_{n}=m$; but $E d_{1}=\ldots=E d_{n}$, hence $n E d_{i}=m$ and $E d_{i}=m / n$.
} 
Table 1 For specified numbers of sellers and buyers, the last column displays expected output per unit of capacityor, equivalently, each buyer's service probability-when sellers are visited with the same probability by any buyer

\begin{tabular}{rrrl}
\hline$n$ & $m$ & $m / n$ & $E y_{i} / \bar{y}_{i}$ \\
\hline 2 & 2 & 1 & 0.750 \\
2 & 100 & 50 & 0.960 \\
2 & 200 & 100 & 0.972 \\
4 & 4 & 1 & 0.683 \\
4 & 200 & 50 & 0.951 \\
4 & 400 & 100 & 0.965 \\
10 & 10 & 1 & 0.651 \\
10 & 500 & 50 & 0.947 \\
10 & 1000 & 100 & 0.962 \\
\hline
\end{tabular}

A simple exercise of comparative statics will be instructive. For any given number of sellers $n$, let the number of buyers $m$-and hence total demand-increase while retaining the equality between total demand and total capacity, so that total capacity $m$ and each firm's capacity $m / n$ increase too. By the law of large numbers, the demand $d_{i}$ forthcoming to each firm converges in probability to its expected level $m / n$ as $m$ goes to infinite. As a consequence, given $n, \frac{E y_{i}}{\bar{y}_{i}}$ is the higher as $m$ is the higher and converges to 1 as $m$ goes to infinite. However, as illustrated by Table 1, $\frac{E y_{i}}{\bar{y}_{i}}$ is still significantly lower than 1 , even for quite high levels of $m / n$ : in other words, the inefficiency deriving from buyers' random choice is far from negligible even for quite high levels of $m / n$.

Suppose now instead that the buyers are involved in a multi-stage BS: each buyer demands repeatedly one unit of the commodity. Rather than playing in each stage the MSE of the static BS, the buyers might adhere to a norm of CL. In the context here under scrutiny, such a norm stipulates that, in each stage of the dynamic BS, a buyer who was served in the previous stage be loyal in the next stage and that a buyer who was rationed visit with the same probability any of the sellers she has not yet tried thus far, so long as there are any. (In the first stage, each buyer visits any seller with probability $1 / n$.) It was proved (see Proposition 3 in De Francesco 2005) that if all buyers are constantly adhering to CL, then with unit probability each seller will receive the stable custom of $m / n$ buyers from stage $\tau=\left(m-\bar{y}_{i}+1\right)(n-2)+2$ onwards so that, with unit probability, all buyers will be served and all firms will produce at full capacity from stage $\tau$ onwards. ${ }^{8}$ This means an increase in efficiency compared to the case in which all buyers are playing repeatedly the MSE of the static BS: as indirectly illustrated by Table 1, such an improvement remains significant, even for quite large levels of $m / n{ }^{9}$

\footnotetext{
${ }^{8}$ Of course, the equation for $\tau$ gives $\tau=2$ in our setting in which $m=n=2$ and $\bar{y}_{i}=1$.

${ }^{9}$ For instance, with $n=10$ sellers and $m=500$ buyers, the probability of being served is 0.947 under repeated playing of the MSE of the static BS. This means that, on the other hand, CL eventually leads to an increase in service probability by $5.3 \%$ compared to persistent randomization.
} 
Let us come back to our two-seller, two-buyer, and two-stage BS setup. Given the benefits of CL in terms of the certainty of obtaining the good in the final stage of the $\mathrm{BS}$, one can expect that an equilibrium strategy for the BS that incorporates such a norm does exist unless prices differ to such an extent so as to make each buyer's overall prospects - in terms of the price to be paid if served and the probability of being served-definitely better when addressing his own demand to the cheapest seller. The questions to be answered when characterizing such an equilibrium strategy are, first, how precisely the buyer's choice in $t=1$ depends on prices and, second, how close prices have to be in order for CL to be a mutual best response in $t=2$. As will be proved later on, the equilibrium strategy incorporating CL, to be denoted by $\Theta_{h}^{* *}$ (resp., $\Theta_{k}^{* *}$ ) with reference to buyer $h$ (resp., $k$ ) is characterized as follows.

Definition $2 \Theta_{h}^{* *}=\left(v_{h, 1}^{* *}, v_{h, 2}^{* *}\right)$, where

$$
v_{h, 1}^{* *}=v^{* *}\left(p_{A}, p_{B}\right)= \begin{cases}1 & \text { if } p_{A}<\frac{3 p_{B}-1}{2} \\ \widetilde{v} \in[0,1] & \text { if } \frac{3 p_{B}-1}{2} \leq p_{A} \leq \frac{1+2 p_{B}}{3} \\ 0 & \text { if } p_{A}>\frac{1+2 p_{B}}{3}\end{cases}
$$

with

$$
\widetilde{\widetilde{v}}=\widetilde{\widetilde{v}}\left(p_{A}, p_{B}\right)=\left\{\begin{array}{cl}
\frac{1+2 p_{B}-3 p_{A}}{2-p_{A}-p_{B}}=1 & \text { if } \frac{3 p_{B}-1}{2}=p_{A}<\frac{1+2 p_{B}}{3} \\
\frac{1+2 p_{B}-3 p_{A}}{2-p_{A}-p_{B}} \in(0,1) & \text { if } \frac{3 p_{B}-1}{2}<p_{A}<\frac{1+2 p_{B}}{3} \\
\frac{1+2 p_{B}-3 p_{A}}{2-p_{A}-p_{B}}=0 & \text { if } \frac{3 p_{B}-1}{2}<p_{A}=\frac{1+2 p_{B}}{3} \\
\frac{1}{2} & \text { if } \quad p_{A}=p_{B}=1,
\end{array}\right.
$$

and where

$$
v_{h, 2}^{* *}=v_{h, 2}^{* *}\left(I_{h}\right)= \begin{cases}1 & \text { if } \mathbf{e}_{h, 1} \in\left\{A s_{h, 1}, B r_{h, 1}\right\} \text { and } 2 p_{B}-1<p_{A}<\frac{1+p_{B}}{2}, \\ 1 & \text { if } p_{A} \leq 2 p_{B}-1<1 \\ 1 & \text { if } p_{A}<p_{B}=1 \\ 0 & \text { if } \mathbf{e}_{h, 1} \in\left\{B s_{h, 1}, A r_{h, 1}\right\} \text { and } 2 p_{B}-1<p_{A}<\frac{1+p_{B}}{2}, \\ 0 & \text { if } \frac{1+p_{B}}{2} \leq p_{A}<1 \\ 0 & \text { if } p_{A}=1>p_{B} \\ \frac{1}{2} & \text { if } p_{A}=p_{B}=1\end{cases}
$$

$\Theta_{k}^{* *}=\left(v_{k, 1}^{* *}, v_{k, 2}^{* *}\right)$, with $v_{k, 1}^{* *}=v^{* *}\left(p_{A}, p_{B}\right)$ (see Eqs. (19) and (20)) and $v_{k, 2}^{* *}=v_{k, 2}^{* *}\left(I_{h}\right)$, where, similarly to $v_{h, 2}^{* *}\left(I_{h}\right)$, 


$$
v_{k, 2}^{* *}=v_{k, 2}^{* *}\left(I_{h}\right)= \begin{cases}1 & \text { if } \mathbf{e}_{k, 1} \in\left\{A s_{k, 1}, B r_{k, 1}\right\} \text { and } 2 p_{B}-1<p_{A}<\frac{1+p_{B}}{2}, \\ 1 & \text { if } p_{A} \leq 2 p_{B}-1<1 \\ 1 & \text { if } p_{A}<p_{B}=1, \\ 0 & \text { if } \mathbf{e}_{k, 1} \in\left\{B s_{k, 1}, A r_{k, 1}\right\} \text { and } 2 p_{B}-1<p_{A}<\frac{1+p_{B}}{2}, \\ 0 & \text { if } \frac{1+p_{B}}{2} \leq p_{A}<1 \\ 0 & \text { if } p_{A}=1>p_{B} \\ \frac{1}{2} & \text { if } p_{A}=p_{B}=1 .\end{cases}
$$

Remark 2 Since the definition above is quite cumbersome, reviewing it in plain language will be helpful when it comes to prove that the strategy profile $\left(\Theta_{h}^{* *}, \Theta_{k}^{* *}\right)$ is an equilibrium for the dynamic BS. According to the first and the fourth line of Eqs. (21) and (22) $\Theta_{h}^{* *}$ and $\Theta_{k}^{* *}$ prescribe CL if and only if prices are sufficiently close to each other so as to meet system (6). According to the remaining lines, if that system does not hold, then: with $p_{A} \neq p_{B}$, the cheapest seller has to be chosen in $t=2$; with $\left(p_{A}, p_{B}\right)=(1,1)$, the stipulated prescription is to pick either seller with probability $1 / 2 .{ }^{10}$

Consider now the prescriptions of $\Theta_{h}^{* *}$ and $\Theta_{k}^{* *}$ for $t=1$. According to the second line of Eqs. (19) and (20), in order for either seller to be chosen with strictly positive probability-i.e., in order for $\widetilde{\widetilde{v}}\left(p_{A}, p_{B}\right) \in(0,1)$ - prices have to be close enough so as to meet both inequalities in system

$$
\frac{3 p_{B}-1}{2} \leq p_{A} \leq \frac{1+2 p_{B}}{3},
$$

as strict inequalities, for otherwise the cheapest seller has instead to be chosen. The interval $\left[\frac{3 p_{B}-1}{2}, \frac{1+2 p_{B}}{3}\right]$ is non-empty since $p_{B} \leq 1$ and degenerates to 1 if $p_{B}=1$ : indeed,

$$
p_{B}<1 \Longleftrightarrow \frac{3 p_{B}-1}{2}<\frac{1+2 p_{B}}{3}<1,
$$

and

$$
p_{B}=1 \Longleftrightarrow \frac{3 p_{B}-1}{2}=\frac{1+2 p_{B}}{3}=1 .
$$

Notice, furthermore, that system (23) is more restrictive than system (5) whenever $p_{B}<1$ : indeed,

$$
p_{B}<1 \Longleftrightarrow 2 p_{B}-1<\frac{3 p_{B}-1}{2}<\frac{1+2 p_{B}}{3}<\frac{1+p_{B}}{2}<1 .
$$

\footnotetext{
${ }^{10}$ One might as well stipulate that CL applies even with $\left(p_{A}, p_{B}\right)=(1,1)$ (see footnote 16$)$.
} 
If, instead, $p_{B}=1$, then system $\frac{3 p_{B}-1}{2} \leq p_{A} \leq \frac{1+2 p_{B}}{3}$ is equivalent to system $2 p_{B}-1 \leq p_{A} \leq \frac{1+p_{B}}{2}$ and implies $p_{A}=\stackrel{2}{1}$ :

$$
p_{B}=1 \Longleftrightarrow 2 p_{B}-1=\frac{3 p_{B}-1}{2}=\frac{1+2 p_{B}}{3}=\frac{1+p_{B}}{2}=1 .
$$

Consequently, if the system of inequalities (23) holds then: with $p_{B}<1$, at least one of the two inequalities (23) is strict and we have that system (6) a fortiori holds and $p_{A}<1$; with $p_{B}=1$, system (23) is equivalent to system (5) and $p_{A}=1$. Next, let us see why, according to equation (20), firm $A$ has to be visited with probability $\widetilde{\widetilde{v}}\left(p_{A}, p_{B}\right)=\frac{1+2 p_{B}-3 p_{A}}{2-p_{A}-p_{B}}$ in $t=1$ when $\frac{3 p_{B}-1}{2}<p_{A} \leq \frac{1+2 p_{B}}{3}$ or $\frac{3 p_{B}-1}{2} \leq p_{A}<\frac{1+2 p_{B}}{3}$. As one can easily verify, this value is in fact the unique solution in $v_{h, 1}$ of the equation

$$
\begin{aligned}
& \pi\left(A s_{k, 1}\left(v_{h, 1}, 1\right)\right) 2\left(1-p_{A}\right)+\left[1-\pi\left(A s_{k, 1}\left(v_{h, 1}, 1\right)\right)\right]\left(1-p_{B}\right) \\
& \quad=\pi\left(B s_{k, 1}\left(v_{h, 1}, 0\right)\right) 2\left(1-p_{B}\right)+\left[1-\pi\left(B s_{k, 1}\left(v_{h, 1}, 0\right)\right)\right]\left(1-p_{A}\right),
\end{aligned}
$$

where $\pi\left(A s_{k, 1}\left(v_{h, 1}, 1\right)\right)=\frac{v_{h, 1}}{2}+1-v_{h, 1}$ (resp., $\left.\pi\left(B s_{k, 1}\left(v_{h, 1}, 0\right)\right)=v_{h, 1}+\frac{1-v_{h, 1}}{2}\right)$ is the probability that buyer $k$ is served in $t=1$ when $k$ visits $A$ (resp., $B$ ) and $h^{2}$ is visiting $A$ with probability $v_{h, 1}$. Note that, in view of Proposition 2, the LHS (resp., the RHS) of Eq. (28) is buyer $k$ 's payoff over the BS if visiting $A$ (resp., $B$ ) in $t=1$, conditional on buyer $h$ visiting $A$ with probability $v_{h, 1}$ in $t=1$ and on buyers $h$ and $k$ adhering to CL in $t=2$ : in fact, under these circumstances, when visiting $A$ (resp., $B$ ) in $t=1$ buyer $k$ obtains a total surplus of $2\left(1-p_{A}\right)\left(\right.$ resp., $\left.2\left(1-p_{B}\right)\right)$ over the BS if served in $t=1$ and a total surplus of $1-p_{B}$ (resp., $1-p_{A}$ ) if rationed in $t=1$. Thus, $\widetilde{\widetilde{v}}\left(p_{A}, p_{B}\right)=\frac{1+2 p_{B}-3 p_{A}}{2-p_{A}-p_{B}} \in[0,1]$ turns out to be the level of $v_{h, 1}$ which makes buyer $k$ 's payoff (under CL by $h$ and $k$ in $t=2$ ) be the same no matter whether $k$ visits $A$ or $B$ in $t=1$.

There is no meaningful solution to Eq. (28) if system (23) does not hold: under such an event, according to equation (19) the cheapest seller has to be visited in $t=1{ }^{11}$ Notice, finally, that except for a discontinuity at $\left(p_{A}, p_{B}\right)=(1,1),{ }^{12}$ the function $v^{* *}\left(p_{A}, p_{B}\right)$ is continuous over the domain $[0 ; 1]^{2}$ : in fact, with $p_{B}<1, \widetilde{\widetilde{v}}\left(p_{A}, p_{B}\right)$ is continuous for $p_{A} \in\left[\frac{3 p_{B}-1}{2}, \frac{1+2 p_{B}}{3}\right]$ and, furthermore, $v^{* *}\left(p_{A}, p_{B}\right)=1$ for $p_{A} \leq \frac{3 p_{B}-1}{2}$ and $v^{* *}\left(p_{A}, p_{B}\right)=0$ for $p_{A} \geq \frac{1+2 p_{B}}{3}$ (note that $v^{* *}\left(p_{A}, p_{B}\right)=\widetilde{\widetilde{v}}=1$ for $p_{A}=\frac{3 p_{B}-1}{2}$ and $v^{* *}\left(p_{A}, p_{B}\right)=\widetilde{\widetilde{v}}=0$ for $\left.p_{A}=\frac{1+2 p_{B}}{3}\right)$.

We are now in a position to establish the following result.

Proposition 3 The strategy profile $\left(\Theta_{h}^{* *}, \Theta_{k}^{* *}\right)$ induces a Nash equilibrium in each $\left(p_{A}, p_{B}\right)-B S$.

Proof In the Appendix.

\footnotetext{
$\overline{11}$ The cheapest seller has also to be visited in $t=1$ if one of the weak inequalities in system (23) holds as an equality.

$12 v^{* *}(1,1)=\widetilde{\widetilde{v}}(1,1)=1 / 2<\lim _{p_{A}>1} v^{* *}\left(p_{A}, 1\right)=1$.
} 
Since any dynamic $\left(p_{A}, p_{B}\right)$-BS has no proper subgames, any of its Nash equilibria is subgame perfect. Hence one should check that, at information sets off the equilibrium path, a buyer is actually making a best response by adhering to his equilibrium strategy. This check is immediate for the equilibrium $\left(\Theta_{h}^{*}, \Theta_{k}^{*}\right)$ : no matter $\mathbf{e}_{h, 1}$ and what buyer $h$ believes about $k$ 's previous move, by playing $v_{h, 2}=v^{*}$ buyer $h$ is obviously making a best response to $v_{k, 2}=v^{*}$. As for the equilibrium $\left(\Theta_{h}^{* *}, \Theta_{k}^{* *}\right)$, Proposition 4 below will show that, along with a proper system of beliefs, that strategy profile represents an "assessment equilibrium", ${ }^{13}$ in the specific meaning we are now going to specify.

At any information set $I_{h}$, buyer $h$ holds a belief $\mu\left(\mathcal{E}_{k, 1} \mid I_{h}\right)$, namely, an (ex-post) probability distribution over the set of events $\mathcal{E}_{k, 1}$ that buyer $k$ may have faced in $t=1$. This allows buyer $h$ to compute $u_{h, 2}\left(v_{h, 2}, \Theta_{k \mid 2}^{* *} ; \mu\left(\mathcal{E}_{k, 1} \mid I_{h}\right) \mid I_{h}\right)$, namely, his expected surplus for $t=2$, conditional on $I_{h}$, when playing $v_{h, 2}$ and with buyer $k$ adhering to the prescriptions of $\Theta_{k}^{* *}$ in $t=2$. A "structurally consistent" system of beliefs is such that, at any information set $I_{h}$, the belief $\mu\left(\mathcal{E}_{k, 1} \mid I_{h}\right)$ is derived by Bayes' rule and the strategy buyer $k$ is conjectured to have followed in $t=1$. According to Proposition 4 below, for any structurally consistent belief system, the strategy profile $\left(\Theta_{h}^{* *}, \Theta_{k}^{* *}\right)$ is "sequentially rational": at any information set $I_{h}$, by playing $v_{h, 2}=v_{h, 2}^{* *}\left(I_{h}\right)$ buyer $h$ maximizes $u_{h, 2}\left(v_{h, 2}, \Theta_{k \mid 2}^{* *} ; \mu\left(\mathcal{E}_{k, 1} \mid I_{h}\right) \mid I_{h}\right)$.

Proposition 4 The assessment $\left(\Theta_{h}^{* *}, \Theta_{k}^{* *} ; \mu\right)$ - $\mu$ being any structurally consistent system of beliefs-meets sequential rationality in any $\left(p_{A}, p_{B}\right)$-BS.

Proof Sequential rationality is trivial to check for $\left(p_{A}, p_{B}\right)=(1,1)$; it is also immediate if $\frac{1+p_{B}}{2} \leq p_{A}<1$ or $p_{B}<p_{A}=1$ (resp., $p_{A} \leq 2 p_{B}-1<1$ or $p_{A}<p_{B}=1$ ) since then $v_{h, 2}^{* * *}=0$ (resp., $v_{h, 2}^{* *}=1$ ) is obviously a best response, no matter $v_{k, 2}$. Next consider BSs in which system (6) holds. At $I_{h}=\left(A r_{h, 1}, \cdot\right), h$ obviously infers that $k$ was served by $A$ in $t=1$ and hence $k$ is expected to be loyal to $A$ in $t=2$ : therefore, $u_{h, 2}\left(v_{h, 2}, \Theta_{k \mid 2}^{* *} ; \mu\left(\mathcal{E}_{k, 1} \mid\left(A r_{h, 1}, \cdot\right)\right) \mid\left(A r_{h, 1}, \cdot\right)\right)=v_{h, 2}\left(1-p_{A}\right) / 2+\left(1-v_{h, 2}\right) \quad\left(1-p_{B}\right)$, which is maximal for $v_{h, 2}=v_{h, 2}^{* *}\left(A r_{h, 1}, \cdot\right)=0$ since $p_{A}>2 p_{B}-1$. At $I_{h}=\left(A s_{h, 1}, \cdot\right)$, any structurally consistent belief is such that $\mu\left(A r_{k, 1} \mid\left(A s_{h, 1}, \cdot\right)\right)$ $+\mu\left(B s_{k, 1} \mid\left(A s_{h, 1}, \cdot\right)\right)=1{ }^{14}$ Therefore, buyer $k$ is expected to visit seller $B$ in $t=2$ : hence $u_{h, 2}\left(v_{h, 2}, \Theta_{k \mid 2}^{* *} ; \mu\left(\mathcal{E}_{k, 1} \mid\left(A r_{h, 1}, \cdot\right)\right) \mid\left(A r_{h, 1}, \cdot\right)\right)=v_{h, 2}\left(1-p_{A}\right)+\left(1-v_{h, 2}\right)\left(1-p_{B}\right) / 2$, which is maximal for $v_{h, 2}=v_{h, 2}^{* *}\left(A s_{h, 1}, \cdot\right)=1$ since $p_{A}<\frac{1+p_{B}}{2}$. A similar reasoning applies to information sets $\left.I_{h}=\left(B s_{h, 1}, \cdot\right)\right)$ and $I_{h}=\left(B r_{h, 1}, \cdot\right)$.

For any $\left(p_{A}, p_{B}\right)$-BS, we denote by $E y_{A, t}^{*}\left(p_{A}, p_{B}\right)$ and $\sum_{t=1}^{2} E \Pi_{A, t}^{*}\left(p_{A}, p_{B}\right)$ firm $A$ 's (ex ante) stage- $t$ expected output and expected total profit, respectively, conditional on the equilibrium $\left(\Theta_{h}^{*}, \Theta_{k}^{*}\right)$ being played: $E y_{A, t}^{*}\left(p_{A}, p_{B}\right)=E y_{A}\left(v^{*}\left(p_{A}, p_{B}\right)\right)$ for $t=1,2$ (see Proposition 1 and Eqs. (13) and (14)) and hence

\footnotetext{
13 As in De Francesco (2005), we borrow this terminology from Binmore (1992, pp. 536-540) to refer to a weakened version of Kreps and Wilson's (1982) "sequential equilibrium."

14 For instance, let the structurally consistent belief be derived from the conjecture that $k$ has adhered to $\Theta_{k}^{* *}$ in $t=1$. Then, as can easily be verified, Bayes' rule yields $\mu\left(A r_{k, 1} \mid\left(A s_{h, 1}, \cdot\right)\right)=\frac{\widetilde{v}}{2-\widetilde{v}}$ and $\mu\left(B s_{k, 1} \mid\left(A s_{h, 1}, \cdot\right)\right)=\frac{2-2 \widetilde{\tilde{v}}}{2-\widetilde{\tilde{v}}}$ if $\frac{3 p_{B}-1}{2} \leq p_{A} \leq \frac{1+2 p_{B}}{3}, \mu\left(B s_{k, 1} \mid\left(A s_{h, 1}, \cdot\right)\right)=1 \quad$ if $\left(1+2 p_{B}\right) / 3<p_{A}^{2-\widetilde{v}}$ and $\mu\left(A r_{k, 1} \mid\left(A s_{h, 1}, \cdot\right)\right)=1$ if $^{-\widetilde{v}} p_{A}<\left(3 p_{B}-1\right) / 2$.
} 
$\sum_{t=1}^{2} E \Pi_{A, t}^{*}\left(p_{A}, p_{B}\right)=2 p_{A} E y_{A}\left(v^{*}\left(p_{A}, p_{B}\right)\right)$. Similarly, we denote by $\left.E y_{A, t}^{* * *}\left(p_{A}, p_{B}\right)\right)$ and $\sum_{t=1}^{2} E \Pi_{A, t}^{* *}\left(p_{A}, p_{B}\right)$ firm $A$ 's (ex-ante) stage- $t$ expected output and expected total profit, respectively, conditional on the equilibrium $\left(\Theta_{h}^{* *}, \Theta_{k}^{* * *}\right)$ being played; note that $E y_{A, 1}^{* *}\left(p_{A}, p_{B}\right)=E y_{A}\left(v^{* *}\left(p_{A}, p_{B}\right)\right)$ (see Eqs. (14), (19) and (20)).

Again, condition (16) can be taken for granted since $p_{A}=0$ and $p_{A}=1$ are never best responses. This fact relies upon the argument in the previous section when the equilibrium $\left(\Theta_{h}^{*}, \Theta_{k}^{*}\right)$ is assumed to be played. The point is also easy to make under the equilibrium $\left(\Theta_{h}^{* *}, \Theta_{k}^{* *}\right): p_{A}=0$ is not a best response, even with $p_{B}=0$, since $\sum_{t=1}^{2} E \Pi_{A, t}^{* *}\left(p_{A}, 0\right)>0$ for $p_{A}$ sufficiently close to $0 ;{ }^{15}$ and $p_{A}=1$ is not a best response, even with $p_{B}=1$, since $\sum_{t=1}^{2} E \Pi_{A, t}^{* *}(1,1)=\frac{3}{4}+\frac{3}{4}$ $=\frac{3}{2}<\lim _{p_{A}>1} \sum_{t=1}^{2} E \Pi_{A, t}^{* *}\left(p_{A}, 1\right)=2 .{ }^{16}$

We are now ready to address price determination at a subgame perfect equilibrium (SPE) of the entire game. As the next proposition makes clear, equilibrium prices are significantly affected by the equilibrium of the BS that is played by the buyers.

\section{Proposition 5}

(i) $\left(\left(p_{A}, p_{B}\right),\left(\Theta_{h}, \Theta_{k}\right)\right)=\left(\left(p^{*}, p^{*}\right),\left(\Theta_{h}^{*}, \Theta_{k}^{*}\right)\right)$, with $p^{*}=1 / 2$, is a subgame perfect equilibrium (SPE) of the entire game.

(ii) $\left(\left(p_{A}, p_{B}\right),\left(\Theta_{h}, \Theta_{k}\right)\right)=\left(\left(p^{* *}, p^{* *}\right),\left(\Theta_{h}^{* *}, \Theta_{k}^{* *}\right)\right)$, with $p^{* *}=7 / 12$, is another SPE.

\section{Proof}

(i) Recall that $\sum_{t=1}^{2} E \Pi_{A, t}^{*}\left(p_{A}, p_{B}\right)=2 p_{A} E y_{A}\left(v^{*}\left(p_{A}, p_{B}\right)\right)$. Holding system (5), $\partial \sum_{t=1}^{2} E \Pi_{A, t}^{*}\left(p_{A}, p_{B}\right) / \partial p_{A}=2\left[E y_{A}\left(\widetilde{v}\left(p_{A}, p_{B}\right)\right)+\left.p_{A} \frac{d E y_{A}(v)}{d v}\right|_{v=\widetilde{v}\left(p_{A}, p_{B}\right)} \frac{\partial \widetilde{v}\left(p_{A}, p_{B}\right)}{\partial p_{A}}\right]$. At a symmetric equilibrium, $p_{A}=p_{B}=p$ and $v=0.5$ : hence $\partial \sum_{t=1}^{2} E \Pi_{A, t}^{*}\left(p_{A}, p_{B}\right) / \partial p_{A}=0$ yields $p=0.5$, as with the static BS.

(ii) For $p_{B} \in(0,1), \sum_{t=1}^{2} E \Pi_{A, t}^{* *}\left(p_{A}, p_{B}\right)$ can be written as

$$
\sum_{t=1}^{2} E \Pi_{A, t}^{* *}\left(p_{A}, p_{B}\right)= \begin{cases}2 p_{A} & \text { if } p_{A} \in\left[0, \frac{3 p_{B}-1}{2}\right), \\ p_{A} E y_{A}\left(\widetilde{\widetilde{v}}\left(p_{A}, p_{B}\right)\right)+p_{A} & \text { if } p_{A} \in\left[\max \left\{0, \frac{3 p_{B}-1}{2}\right\}, \frac{1+2 p_{B}}{3}\right] \\ p_{A} & \text { if } \quad p_{A} \in\left(\frac{1+2 p_{B}}{3}, \frac{1+p_{B}}{2}\right), \\ 0 & \text { if } p_{A} \in\left[\frac{1+p_{B}}{2}, 1\right],\end{cases}
$$

where the interval $\left[0, \frac{3 p_{B}-1}{2}\right)$ pertaining to the first line on the RHS is empty if and only if $p_{B} \leq 1 / 3$ and the intervals in all subsequent lines are non-empty and non-degenerate since $p_{B}<1$. If $p_{A} \in\left[0, \frac{3 p_{B}-1}{2}\right)$, then $\sum_{t=1}^{2} E \Pi_{A, t}^{* *}\left(p_{A}, p_{B}\right)=2 p_{A}$ since firm $A$ is visited by both buyers in $t=1$ and by at least one buyer in $t=2$

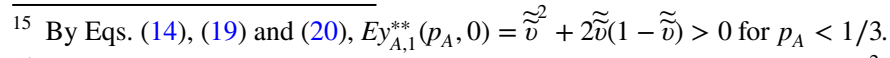

${ }^{16}$ If we had let CL apply also for $\left(p_{A}, p_{B}\right)=(1,1)$, still it would have been $\sum_{t=1}^{2} E \Pi_{A, t}^{* *}(1,1)=\frac{3}{4}+1<2$.
} 


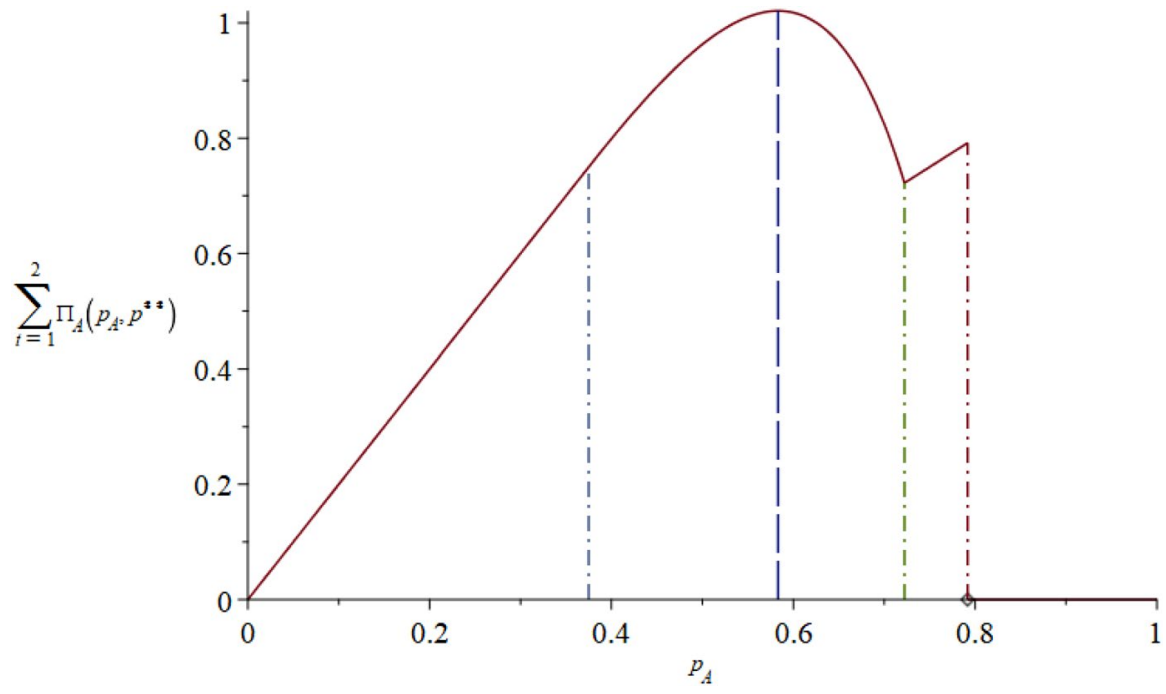

Fig. 1 The curve shows firm $A$ 's payoff as a function of $p_{A}$, for $p_{B}=p^{* *}=7 / 12=0.58 \overline{3}$, conditional on the equilibrium $\left(\Theta_{h}^{* *}, \Theta_{k}^{* *}\right)$ being played in any $\left(p_{A}, p_{B}\right)$-BS

(by both buyers if it is also $p_{A} \leq 2 p_{B}-1$ ). Note that, for $p_{A} \in\left[0, \frac{1+p_{B}}{2}\right)$, $\sum_{t=1}^{2} E \Pi_{A, t}^{* *}\left(p_{A}, p_{B}\right)=p_{A} E y_{A}\left(v^{* * *}\left(p_{A}, p_{B}\right)\right)+p_{A}$ is a continuous function of $p_{A}$. Indeed: $E y_{A, 1}^{* *}\left(\frac{1+2 p_{B}}{3}, p_{B}\right)=E y_{A}\left(\widetilde{\widetilde{v}}\left(\frac{1+2 p_{B}}{3}, p_{B}\right)\right)=0=\lim _{p_{A}}>\frac{1+2 p_{B}}{3} E y_{A, 1}^{* *}\left(p_{A}, p_{B}\right) ; \quad$ with $\quad p_{B} \geq 1 / 3$, $E y_{A, 1}^{* *}\left(\frac{3 p_{B}-1}{2}, p_{B}\right)=E y_{A}\left(\widetilde{\widetilde{v}}\left(\frac{3 p_{B}-1}{2}, p_{B}\right)\right)=1=\lim _{p_{A} \zeta \frac{3 p_{B}-1}{2}} E y_{A, 1}^{* *}\left(p_{A}, p_{B}\right)$. A discontinuity arises at $p_{A}=\frac{1+p_{B}}{2}$ since $\lim _{p_{A} \succ \frac{1+p_{B}}{2}} E y_{A, 2}^{* *}\left(p_{A}, p_{B}\right)=1>E y_{A, 2}^{* *}\left(\frac{1+p_{B}}{2}, p_{B}\right)=0$. For $\quad p_{A} \in\left(\max \left\{0, \frac{3 p_{B}-1}{2}\right\}, \frac{1+2 p_{B}}{3}\right), \quad \frac{\partial \sum_{t=1}^{2} E \Pi_{A, t}^{* *}\left(p_{A}, p_{B}\right)}{\partial p_{A}}=E y_{A}\left(\widetilde{\widetilde{v}}\left(p_{A}, p_{B}\right)\right)$ $+\left.p_{A} \frac{d E y_{A}(v)}{d v}\right|_{v=\widetilde{v}\left(p_{A}, p_{B}\right)} \frac{\partial \widetilde{v}\left(p_{A}, p_{B}\right)}{\partial p_{A}}+1$, which is positive on a right neighbourhood of $\max \left\{0, \frac{3 p_{B}-1}{2}\right\}$ since $\left.p_{A} \frac{d E y_{A}(v)}{d v}\right|_{v=\widetilde{\widetilde{v}}\left(p_{A}, p_{B}\right)}=0$ at $p_{A}=\max \left\{0, \frac{3 p_{B}-1}{2}\right\}$ (note

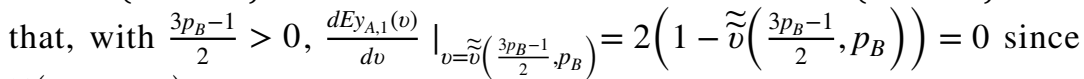
$\left.\widetilde{\widetilde{v}}\left(\frac{3 p_{B}-1}{2}, p_{B}\right)=1\right)$, and negative on a left neighbourhood of $\frac{1+2 p_{B}}{3}$, since $\lim _{p_{A} \zeta \frac{1+2 p_{B}}{3}} \frac{\partial \sum_{t=1}^{2} E \Pi_{A, t}^{* *}\left(p_{A}, p_{B}\right)}{\partial p_{A}}=\frac{-1-17 p_{B}}{5\left(1-p_{B}\right)}<0 .{ }^{17}$ Also, over the range $\left(\max \left\{0, \frac{3 p_{B}-1}{2}\right\}, \frac{1+2 p_{B}}{3}\right), \sum_{t=1}^{2} E \Pi_{A, t}^{* *}\left(p_{A}, p_{B}\right)$ is strictly concave in $p_{A}:{ }^{18}$ thus,

\footnotetext{
17 Note that $\lim _{p_{A} \gamma \frac{1+2 p_{B}}{3}}\left[E y_{A}(v)\right]_{v=\widetilde{v}\left(p_{A}, p_{B}\right)}=0, \lim _{p_{A} \gamma \frac{1+2 p_{B}}{3}} \frac{d E y_{A}(v)}{d v} I_{v=\widetilde{\tilde{v}}\left(p_{A}, p_{B}\right)}=2, \lim _{p_{A} \gamma \frac{1+2 p_{B}}{3}} \frac{\partial \widetilde{\tilde{v}}\left(p_{A}, p_{B}\right)}{\partial p_{A}}=\frac{-9}{5\left(1-p_{B}\right)}$. $18 \frac{\partial^{2} \sum_{t=1}^{2} E \Pi_{A, t}^{* *}\left(p_{A}, p_{B}\right)}{\partial p_{A}^{2}}=\left.2 \frac{d\left(E y_{A}(v)\right)}{d v}\right|_{v=\widetilde{\widetilde{v}}\left(p_{A}, p_{B}\right)} \frac{\partial \widetilde{\tilde{v}}\left(p_{A}, p_{B}\right)}{\partial p_{A}}+\left.p_{A} \frac{d^{2} E y_{A}(v)}{d v^{2}}\right|_{v=\widetilde{\tilde{v}}\left(p_{A}, p_{B}\right)}\left(\frac{\partial \widetilde{\tilde{v}}\left(p_{A}, p_{B}\right)}{\partial p_{A}}\right)^{2}+\left.p_{A} \frac{d E y_{A}(v)}{d v}\right|_{v=\widetilde{v} \tilde{v}\left(p_{A}, p_{B}\right)} \frac{\partial^{2} \widetilde{\tilde{v}}\left(p_{A}, p_{B}\right)}{\partial p_{A}^{2}}$, where $\left.\frac{d\left(E y_{A}(v)\right)}{d v}\right|_{v=\widetilde{\widetilde{v}}\left(p_{A}, p_{B}\right)}=2\left(1-\widetilde{\widetilde{v}}\left(p_{A}, p_{B}\right)\right)>0$ since $\widetilde{\widetilde{v}}\left(p_{A}, p_{B}\right) \in(0,1),\left.\frac{d^{2} E y_{A}(v)}{d v^{2}}\right|_{v=\widetilde{\widetilde{v}}\left(p_{A}, p_{B}\right)}=\frac{d^{2} E y_{A}(v)}{d v^{2}}=-2$, $\frac{\partial \widetilde{\tilde{v}}\left(p_{A}, p_{B}\right)}{\partial p_{A}}=-\frac{5\left(1-p_{B}\right)}{\left(2-p_{A}-p_{B}\right)^{2}}<0$ and $\frac{\partial^{2} \widetilde{\tilde{v}}\left(p_{A}, p_{B}\right)}{\partial p_{A}^{2}}=\frac{-10\left(1-p_{B}\right)}{\left(2-p_{A}-p_{B}\right)^{3}}<0$.
} 
over that range, $\sum_{t=1}^{2} E \Pi_{A, t}^{* *}\left(p_{A}, p_{B}\right)$ has a unique and internal maximum. Finally, $\sum_{t=1}^{2} E \Pi_{A, t}^{* *}\left(p_{A}, p_{B}\right)$ is kinked at $p_{A}=\frac{1+2 p_{B}}{3}\left(\lim _{p_{A} \backslash \frac{1+2 p_{B}}{3}} \frac{\partial \sum_{t=1}^{2} E \Pi_{A, t}^{* *}\left(p_{A}, p_{B}\right)}{\partial p_{A}}=1\right)$ and increasing for $p_{A} \in\left(\frac{1+2 p_{B}}{3}, \frac{1+p_{B}}{2}\right)$. Searching for a symmetric equilibrium, we put $p_{A}=p_{B}$ into the first-order condition for an internal maximum, to obtain $p_{A}=p_{B}=p^{* *}=\frac{7}{12}=0.58 \overline{3}$. In fact, $\arg \max _{p_{A \in[0,1]}} \sum_{t=1}^{2} E \Pi_{A, t}^{* *}\left(p_{A}, p^{* *}\right)=p^{* *}$ : $\lim _{p_{A} \nearrow \frac{1+p^{* *}}{2}} \sum_{t=1}^{2} E \Pi_{A, t}^{* *}\left(p_{A}, p^{* *}\right)=\frac{1+p^{* *}}{2}=0.791 \overline{6}$ $<\sum_{t=1}^{2} E \Pi_{A, t}^{* *}\left(p^{* *}, p^{* *}\right)=49 / 48=1.0208 \overline{3}$ (see Fig. 1$)$.

Remark 3 From Proposition 5 we can see that the outcome of price competition in terms of total welfare and its distribution depends on whether, in $t=2$, the buyers keep on randomizing as they do in $t=1$ or adhere instead to CL. On the equilibrium path of the SPE of the entire game, expected total output over the BS is equal for each firm to $\frac{3}{4} \times 2=\frac{3}{2}$ when the buyers are persistently randomizing; by contrast, under CL capacity is certainly fully utilized in $t=2$ and hence each firm's expected total output is $\frac{3}{4}+1=\frac{7}{4}$. The increase in expected total output under CL clearly means an increase in total welfare. At the same time, some redistribution of welfare from the buyers to the sellers also takes place since the corresponding equilibrium price $p^{* *}$ is significantly higher than the equilibrium price $p^{*}$ obtaining with persistent randomization $\left(p^{* *} / p^{*}=1.1 \overline{6}\right)$ : as a consequence, each firm's equilibrium payoff under CL is approximately 36.1 per cent higher than under persistent randomization: $\frac{\sum_{t=1}^{2} E \Pi_{A, t}^{* * * *}\left(p^{* *}, p^{* *}\right)}{\left.\sum_{t=1}^{2} E \Pi_{A, t}^{*}, p^{*}, p^{*}\right)}=\frac{\frac{7}{12} \times \frac{7}{4}}{\frac{1}{2} \times \frac{3}{2}}=1.36 \overline{1}$. As for the buyers, one can check that the negative effect deriving from the higher equilibrium price under CL slightly outweighs the favourable effect deriving from the higher service probability ( 1 instead of $\frac{3}{4}$ ) in $t=2$ : each buyer's expected total surplus under CL is indeed $\left(1-p^{* *}\right)\left(\frac{3}{4}+1\right)=\frac{5}{12} \times \frac{7}{4}=\frac{35}{48}=0.7291 \overline{6}$, slightly less than $\left(1-p^{*}\right) \frac{3}{4} \times 2=\frac{1}{2} \times \frac{3}{4} \times 2=\frac{3}{4}=0.75$, her expected total surplus under persistent randomization.

The underlying reason for the increase in each firm's market power under CL is worth clarifying. As usual, there are a positive and a negative component in firm $A$ 's marginal payoff. To fix ideas, let $p_{A}$ increase. The positive component is the "direct" effect of the price increase, namely, the rate of change of $A$ 's revenue if neglecting the fall in expected output deriving from the price increase; the negative component is the "indirect" effect, namely, the rate of change of $A$ 's revenue deriving from the fall in $A$ 's expected total output over the BS. Starting from a uniform pair of prices, we have that $A$ 's marginal payoff is

$$
\begin{aligned}
\left.\frac{\partial \sum_{t=1}^{2} E \Pi_{A, t}^{* *}\left(p_{A}, p_{B}\right)}{\partial p_{A}}\right|_{\left(p_{A}, p_{B}\right)=(p, p)} & =\left[E y_{A}(v)\right]_{v=1 / 2}+1+\left.\frac{\left.d E y_{A} \widetilde{\widetilde{v}}\left(p_{A}, p_{B}\right)\right)}{d p_{A}}\right|_{\left(p_{A}, p_{B}\right)=(p, p)} \\
& =\left[E y_{A}(v)\right]_{v=1 / 2}+1+\left.\left.p \frac{d E y_{A}(v)}{d v}\right|_{v=1 / 2} \frac{\partial \widetilde{v}\left(p_{A}, p_{B}\right)}{\partial p_{A}}\right|_{\left(p_{A}, p_{B}\right)=(p, p)} \\
& =\left(\frac{3}{4}+1\right)+\left(-\frac{5}{4} \frac{p}{1-p}\right),
\end{aligned}
$$


under the equilibrium $\left(\Theta_{h}^{* *}, \Theta_{k}^{* *}\right)$ of the BS while it is equal to

$$
\begin{aligned}
\left.\frac{\partial \sum_{t=1}^{2} E \Pi_{A, t}^{*}\left(p_{A}, p_{B}\right)}{\partial p_{A}}\right|_{\left(p_{A}, p_{B}\right)=(p, p)} & =2\left[E y_{A}(v)\right]_{v=1 / 2}+\left.2 p \frac{d E y_{A}\left(\widetilde{v}\left(p_{A}, p_{B}\right)\right)}{d p_{A}}\right|_{\left(p_{A}, p_{B}\right)=(p, p)} \\
& =2\left[E y_{A}(v)\right]_{v=1 / 2}+\left.\left.2 p \frac{d E y_{A}(v)}{d v}\right|_{v=1 / 2} \frac{\partial \widetilde{v}\left(p_{A}, p_{B}\right)}{\partial p_{A}}\right|_{\left(p_{A}, p_{B}\right)=(p, p)} \\
& =\left(2 \times \frac{3}{4}\right)+\left(-\frac{3}{2} \frac{p}{1-p}\right),
\end{aligned}
$$

under the equilibrium $\left(\Theta_{h}^{*}, \Theta_{k}^{*}\right)$. Notice that both the positive and the negative components are higher in Eq. (30) than in Eq. (31): $\frac{3}{4}+1>2 \times \frac{3}{4}$ and $-\frac{5}{4} \frac{p}{1-p}>-\frac{3}{2} \frac{p}{1-p}$. This is a consequence of the fact that, under the equilibrium $\left(\Theta_{h}^{* *}, \Theta_{k}^{* *}\right)$, capacity is fully utilized in $t=2$, even when $p_{A}$ is marginally raised above $p_{B}$. In particular, as for the negative component, while the increase of $p_{A}$ has a stronger impact on $A$ 's expected output in $t=1$ under the equilibrium $\left(\Theta_{h}^{* *}, \Theta_{k}^{* *}\right)$ than under the equilibrium $\left(\Theta_{h}^{*}, \Theta_{k}^{*}\right)$-in fact, $\left.\frac{\left.d E y_{A} \widetilde{\widetilde{v}}\left(p_{A}, p_{B}\right)\right)}{d p_{A}}\right|_{\left(p_{A}, p_{B}\right)=(p, p)}=-\frac{5}{4(1-p)}<\left.\frac{d E y_{A}\left(\tilde{v}\left(p_{A}, p_{B}\right)\right)}{d p_{A}}\right|_{\left(p_{A}, p_{B}\right)=(p, p)}=-\frac{3}{4(1-p)}, 19$ the total impact on $A$ 's expected output over the two stages of the BS is nevertheless lower: $\left.\quad \frac{\left.d E y_{A} \widetilde{\widetilde{v}}\left(p_{A}, p_{B}\right)\right)}{d p_{A}}\right|_{\left(p_{A}, p_{B}\right)=(p, p)}=-\frac{5}{4(1-p)}>\left.2 \frac{\left.d E y_{A} \widetilde{v}\left(p_{A}, p_{B}\right)\right)}{d p_{A}}\right|_{\left(p_{A}, p_{B}\right)=(p, p)}=2 \times\left(-\frac{3}{4(1-p)}\right)$ and this is because the price increase does not affect $A$ 's output in $t=2$. Thus, at any uniform price pair $(p, p)$, the incentive to unilaterally increase the price is higher under the equilibrium $\left(\Theta_{h}^{* *}, \Theta_{k}^{* *}\right)$; as a consequence, since at $\left(p_{A}, p_{B}\right)=\left(p^{*}, p^{*}\right)$ there is no incentive to unilaterally deviate if the equilibrium $\left(\Theta_{h}^{*}, \Theta_{k}^{*}\right)$ of the BS is played, at that same price pair there is an incentive to unilaterally increase the price if the equilibrium $\left(\Theta_{h}^{* *}, \Theta_{k}^{* *}\right)$ of the BS is played: $\left.\frac{\partial \sum_{t=1}^{2} E \Pi_{A, t}^{* *}\left(p_{A}, p_{B}\right)}{\partial p_{A}}\right|_{\left(p_{A}, p_{B}\right)=\left(p^{*}, p^{*}\right)}$ $=\left.\frac{\partial \sum_{t=1}^{2} E \Pi_{B, t}^{* *}\left(p_{A}, p_{B}\right)}{\partial p_{B}}\right|_{\left(p_{A}, p_{B}\right)=\left(p^{*}, p^{*}\right)}>0$.

\section{Conclusion}

The main message arising from our findings is that the outcome of price competition among capacity constrained sellers may depend to some extent on whether the buyers' decisions are made just once or repeatedly after the setting of prices. Compared to previous analyses of Bertrand-Edgeworth competition under imperfect mobility and imperfect information on the part of the buyers, this paper has modelled a situation where the buyers demand a certain commodity repeatedly over the time horizon in which prices are fixed, each time choosing which of the existing sellers to try. Reconsidering buyers' incentives within a dynamic BS has delivered neat results in a two-seller, two-buyer and two-stage BS setup. Firstly, whenever price differences at the sellers are sufficiently small or non-existent, another equilibrium exists

\footnotetext{
19 This is because $\widetilde{\widetilde{v}}\left(p_{A}, p_{B}\right)$ falls more than $\widetilde{v}\left(p_{A}, p_{B}\right)$ does when $p_{A}$ increases above $p_{B}$. In fact, as the reader can easily check by comparing Eqs. (4) and (20), $\widetilde{v}\left(p_{A}, p_{B}\right) \lesseqgtr \widetilde{v}\left(p_{A}, p_{B}\right)$ according as to whether $p_{A} \gtreqless p_{B}$.
} 
for the dynamic BS, besides that in which the buyers play repeatedly the MSE of the static BS: in this alternative equilibrium, the buyers are conditionally loyal to the seller previously chosen, which in its turn ensures that, in the second stage of the BS, both buyers are served and the capacity of both sellers is fully exploited. In such a way, conditional loyalty proves to be an effective (ex-post) coordination device for the buyers: through conditional loyalty, a perfect matching - that would obtain any time under buyers' perfect mobility - will certainly be reached by the last stage of a two-stage BS. In a sense, through conditional loyalty the buyers learn how to play, in the last stage, one of the two PSEs of the static BS obtaining when prices are sufficiently close to each other. Quite remarkably, it pays to adhere to conditional loyalty even though, in the present model, the sellers do not give any service priority to loyal customers and each buyer has only imperfect information on the other buyer's previous move: ${ }^{20}$ even when-as it is the case, for instance, at the equilibrium of the price game- the two sellers are charging the same price, in the final stage of the BS a buyer is not "indifferent" among the two sellers if the other buyer adheres to conditional loyalty. Secondly, the equilibrium of the BS exhibiting conditional loyalty positively affects each firm's market power: at the corresponding equilibrium of the price game the uniform price is significantly higher than the equilibrium price arising when, in each stage of the dynamic BS, the buyers are playing the MSE of the static BS. Consequently, conditional loyalty leads to a remarkable increase in each seller's payoff while leading to a slight decrease in each buyer's payoff.

At this point, the question naturally arises as to the generality of the foregoing results. Although further research is warranted to provide a definite answer, preliminary findings from earlier work suggest that some basic results should extend beyond the simple setup envisaged in this paper. First of all, and as already recalled in Remark 1, in the presence of several buyers and sellers, conditional loyalty remains a powerful coordination device in the sense that mismatchings between demand and capacity at the firm's level will be corrected in a finite number of stages. As illustrated by Table 1 above, this may still represent a significant increase in efficiency compared to the repeated play of the MSE of the static BS. At the same time, characterizing a buyer's strategy that, when prices are sufficiently close at the different sellers, calls for conditional loyalty from some stage of a dynamic BS onwards and that, along with consistent beliefs, constitutes an "assessment equilibrium", is a more difficult task to accomplish in a context in which several sellers are facing several buyers who are involved in a several-stage BS after the setting of prices.

A final question is how, in a more general setup, the common adoption of a strategy incorporating conditional loyalty would affect the pricing decisions of the sellers compared to the case in which the buyers are playing repeatedly the MSE of the static BS. On the one hand, quite intuitively, conditional loyalty should again result in some increase in equilibrium prices whenever, as in the present paper, the number and the capacity of sellers are fixed and the time horizon of the buyers' decisions is made up of a finite number of stages of a BS over which prices are fixed. On the other hand,

\footnotetext{
${ }^{20}$ Note that information sets $I_{h}=\left(A s_{h, 1}, \cdot\right)$ do not fully reveal $k$ 's previous action to buyer $h$ since they are consistent with $A r_{k, 1}$ as well as $B s_{k, 1}$. (An analogous remark applies to information sets $I_{h}=\left(B s_{h, 1}, \cdot\right)$.)
} 
the picture is likely to change significantly if the industry is open to entry: when the possibility of entry is taken into account, it seems reasonable to expect that it is the buyers who benefit the most from the increased efficiency brought about by conditional loyalty.

Supplementary Information The online version contains supplementary material available at https://doi. org/10.1007/s43546-021-00142-w.

Funding Open access funding provided by Università degli Studi di Siena within the CRUI-CARE Agreement.

Data availability All data analysed are included in the paper.

Code availability Not applicable.

\section{Declarations}

Conflict of interest The author declares that there is no conflict of interest regarding this article.

Open Access This article is licensed under a Creative Commons Attribution 4.0 International License, which permits use, sharing, adaptation, distribution and reproduction in any medium or format, as long as you give appropriate credit to the original author(s) and the source, provide a link to the Creative Commons licence, and indicate if changes were made. The images or other third party material in this article are included in the article's Creative Commons licence, unless indicated otherwise in a credit line to the material. If material is not included in the article's Creative Commons licence and your intended use is not permitted by statutory regulation or exceeds the permitted use, you will need to obtain permission directly from the copyright holder. To view a copy of this licence, visit http://creativecommons.org/licen ses/by/4.0/.

\section{References}

Binmore K (1992) Fun and games. D. C. Heath, Lexington

Burdett K, Shi S, Wright R (2001) Pricing and matching with frictions. J Political Econ 109:1060-1085. https://doi.org/10.1086/322835

Camera G, Selcuk C (2009) Price dispersion with directed search. J Eur Econ Assoc 7:1193-1224. https:// doi.org/10.1162/JEEA.2009.7.6.1193

De Francesco MA (1998) The emergence of customer markets in a dynamic buyer game. Quaderni del Dipartimento di Economia Politica, Working Paper 225, Siena

De Francesco MA (2005) Matching buyers and sellers. Econ Bull 3(31):1-10

Deneckere R, Peck J (1995) Competition over price and service rate when demand is stochastic: a strategic analysis. RAND J Econ 26:148-162. https://doi.org/10.2307/2556040

Geromichalos A (2014) Directed search and the Bertrand paradox. Int Econ Rev 55:1043-1065. https:// doi.org/10.1111/iere.12082

Goldman CV, Kraus S, Shehory O (2004) On experimental equilibria strategies for selecting sellers and satisfying buyers. Decis Support Syst 38:329-346. https://doi.org/10.1016/S0167-9236(03)00119-2

Kreps DM, Wilson R (1982) Sequential equilibria. Econometrica 50:863-894. https://doi.org/10.2307/ 1912767

Peters M (1984) Bertrand equilibrium with capacity constraints and restricted mobility. Econometrica 52:1117-1128. https://www.jstor.org/stable/i332677

Peters M (2000) Limits of exact equilibria for capacity constrained sellers with costly search. J Econ Theory 95:139-168. https://doi.org/10.1006/jeth.2000.2667

Shi S (2016) Customer relationship and sales. J Econ Theory 166:483-516. https://doi.org/10.1016/j.jet. 2016.09.012 